\title{
Factor Influencing Decision for Eye Screening among Diabetes Patients: A Review
}

\author{
Mat Isa $\mathbf{R}^{1}$, Saidi $\mathbf{S}^{1}$, Salam $\mathbf{A}^{2}$ and Sumailyah Jamaludin TS*1 \\ ${ }^{1}$ Kulliyyah of Nursing, International Islamic University Malaysia, Malaysia \\ ${ }^{2}$ Kulliyyah of Medicine, International Islamic University Malaysia, Malaysia
}

*Corresponding author: Thandar Soe Sumaiyah Jamaludin, Kulliyyah of Nursing, International Islamic University Malaysia, Malaysia, Tel: +609-57072667; Email: sumaiyah@ iium.edu.my

\section{Research Article \\ Volume 4 Issue 1}

Received Date: January 06, 2020

Published Date: February 07, 2020

DOI: $10.23880 /$ hij-16000215

\section{Abstract}

Introduction: Individuals with Diabetes Mellitus (DM) are at increased risk for developing diabetic ocular complications.

Materials and Methods: A systematic approach was applied to find the relevant topic regarding the said issue. This literature review aimed to determine the system of eye screening, reasons for seeking eye screening and their treatment.

Results: A total of 22 papers and one guideline were reviewed in this study. There were four main barriers found in this study regarding with factor influencing decision for eye screening among diabetes patients. Those were diabetic eye screening, management issues on diabetic eye screening and barriers to uptake diabetic eye screening. The details of these finding results were discussed further in the discussion section.

Conclusion: The health care system, health care provider and patients itself could provide a clear explanation of factors influencing the decision for diabetic eye screening. A further study is recommended to validate these review findings.

Keywords: Eye screening; Diabetes mellitus; Diabetic retinopathy

Abbreviations: DM: Diabetes Mellitus; WHO: World Health Organization; DR: Diabetic retinopathy; NADI: National Diabetes Institute; BIO: Binocular Indirect Ophthalmoscope; MO: Medical Officer; STDR: Sight-Threatening Diabetic Retinopathy; UMMC: University Malaya Medical Centre; PDR: Proliferative Diabetic Retinopathy; HCP: Health Care Professionals.

\section{Introduction}

Diabetes Mellitus (DM) is on a rising trend globally. The World Health Organization (WHO) has estimated that in year 2030, Malaysia would have a total of 24.8 million people with DM. In Malaysia, based on the National Health and Morbidity
Survey 2011, the prevalence of known and newly diagnosed diabetes has raised from $11.6 \%$ in 2006 to $15.3 \%$ in 2011 from age above 18 and 30 years old respectively. Diabetic retinopathy (DR) is one of the most common diabetes complications which affected $36.8 \%$ of diabetes patients in Malaysia.

\section{Methods}

The review methodology used systematic approach. This study aimed to identify literature which gives evidence of a predefined content and quality that required to be discussed further. A pragmatic approach to the review was therefore adopted, primarily to enable a focused and technically 
manageable overview. The precise inclusion and exclusion criteria, and search strategies are as followed.

\section{Study Eligibility Criteria}

The inclusion criteria were the report was written in English, the report carried data about adults aged $\geq 18$ years' old and adult patients with Diabetes Mellitus Type I and type II. While the exclusion criteria were studies about DM patients $<18$ years' old.

\section{Search Strategy}

The literature review was conducted as a systematic approach between September 2015 to February 2016 in order to summarise, synthesize and draw conclusion about a topic based on the evidence from the included studies. The search strategy used the terms 'diabetes mellitus', 'diabetes complication', 'diabetic ocular', 'eye screening'. Subsequently, the search expands by linking the initial keywords including 'adult'. The results of each category were combined using Boolean terms 'AND' or 'OR' to narrow down the search findings. The search limited to English language articles within timeframe from 2005 until January 2017.

\section{Databases}

Electronic database search that are relevant was done on IIUM online database; Springer link, ProQuest, Medscape, and Mosby nursing consult and Ovid-Medline. Besides, information regarding diabetes mellitus database were mainly achieved via email to National Diabetes Institute (NADI) and also from an online clinical database hosted at the Association of Clinical Registry, Malaysia website at www.acrm.org.my/ned.

\section{Result}

Total of 22 papers and one guideline was chosen to be discussed in this study regarding eye screening issues. Most of the study was done at America $(n=3)$, United Kingdom $(n=3)$, Spain $(n=2)$, Ghana $(n=1)$, Oman $(n=1)$, Nigeria $(n=1)$, Korea $(n=1)$, India $(n=1)$, Singapore $(n=1)$, and Malaysia $(n=9)$. Thus, among 22 studies selected, the design were quantitative $(n=3)$, literature review $(n=5)$, systematic review $(n=1)$ and qualitative $(n=3)$.

\section{Participant Characteristics}

Participants in the selected studies were age 18 years old and above. The proportion of male and female in all of these studies were stated, however detail on gender were not included in six studies on systematic review (1), literature review (2-3), meta-analysis study (4-5) and narrative review (6). Overall studies included diabetes mellitus patients and various health care professionals.

\section{Discussion}

\section{Diabetic Eye Screening}

Prevalence of diabetic retinopathy: Diabetic retinopathy (DR) is a leading cause of vision loss worldwide. In 2030, it is estimated that 191.0 million to 56.3 million people will affected with DR. A study done by Rajiv, et al. stated that major risk factors for DR were age, longer duration of disease, neuropathy, urban residence, higher socioeconomic, use of aspirin, men, hypertension and use of insulin. In contrast, Daniel et al, 2016 divided risk factors into modifiable; HbA1c, systolic blood pressure, hyperlipidemia, and body mass index and non-modifiable; puberty and pregnancy. Similarly, both of them agreed that genetic risk factor may influence the incidence of DR but this field is still in its infancy.

Thevi, et al. [1] studied about prevalence of eye disease and visual impairment among the rural population in Temerloh, Pahang. Out of 1081 patients, 275 diabetic patients screened for diabetic retinopathy, 78 patients have changes of diabetic retinopathy, 53 have non-proliferative retinopathy and fifteen patients have proliferative retinopathy. Cataract was the most common disease responsible for severe visual impairment (83.3\%) while for blindness (74.3\%). This study includes new patients that came to eye clinic and not merely focus on diabetic patients.

However, Hussein, et al. was narratively discussed the current status of diabetes in Malaysia. It includes epidemiology, complications lifestyle, pharmacologic treatments and technological approach. This study provides overall information of diabetes management in Malaysia. Likewise, Mafauzy, et al. report on status of Diabetes Control in Malaysia using cross-sectional, observational study. This study found that age and duration of disease were significant predictors of diabetes mellitus complications. This report informed that diabetes complications imply worrisome condition. Thus, further exploration need to evaluate the effectiveness of implementation.

In addition, only few studies discussed regarding the prevalence of attendance to eye screening. A study done by Goh, et al. $[2,3,4]$ stated that the pattern of distribution for the duration of the last eye examination was similar across all age groups, ethnicities and genders. The highest proportion (50.3\%) of those who had previously undergone eye examinations were patients who had been attending government health care center compared to who seek treatment in private and self-medicated. Thus, it represents the low percentage of diabetics who undergone eye examination which represents $32.8 \%$ from 34,539 
respondents who went for yearly eye screening as reported in the National Health and Morbidity Survey 2006.

Awareness of diabetic retinopathy: Two studies were done by Addoor, et al. [5] and Tajunisah, et al. [6] discussed about awareness issue among diabetes patients who give first visit to eye clinic. In the study at Malacca among 351 diabetic patients, Addoor, et al. [5] found that although 91.8\% of the patients with retinopathy were aware of an ocular complication of diabetes but $31.4 \%$ of them having retinopathy and $42.7 \%$ of these patients never had an ophthalmological evaluation. This study found that, duration of diabetes more than 11 years and treatment of insulin have significant effect on the presence of retinopathy.

In addition, $79.8 \%$ were aware of complication of Diabetes Mellitus (DM), 87.2\% knew about disease will effect on eyes but only $50 \%$ underwent ophthalmologist checkup. It concludes that awareness was good but motivation to undergo assessment was poor. And also, peripheral center screening of the diabetes population help the patients to have an insight into their problem. However, this study did not explore on feasibility of health education and health care system. This is clearly indicates that, though the patients were aware about diabetic eye complication, they are not willing or not serious enough to undergo an examination of their eyes. This lack of motivation was not associated with the educational level of the patients nor was associated with the ethnicity of the patients as this study was done at a diabetes clinic, it may reflect diabetes population in Malaysia.

However, in other study done by Tajunisah, et al. [6] was done at eye clinic among type II diabetic patients attending a tertiary medical Centre in Kuala Lumpur, Malaysia. Almost $86 \%$ of respondents aware of diabetic eye complications especially who had achieved tertiary educational level but ethnicity and gender did not relate to awareness. Instead of a high level of awareness, it was unrelated to their selfmotivation but their attendance influence by their doctor referral. However, participants in this study were informed about eye complications because this is their first visit to the eye clinic.

\section{Management Issues on Diabetic Eye Screening}

Eye screening is an important measure for detection and prompt treatment for prevention of diabetes-related visual impairment. Diabetic Retinopathy (DR) screening is performed through assessment of patients' best corrected visual acuity by Snellen chart, using direct ophthalmoscope, dilated slit lamp bio microscopy with a handheld lens (90 D or $78 \mathrm{D}$ ), mydriatic or non-mydratic retinal photography, tele retinal screening and retinal video recording [7].
The objective of assessment of the optical quality of the eye is the great interest in clinical practice. Ocular diffraction, aberrations and scattering influence intraocular retinal image quality, therefore affecting the visual performance of the subject. In human eyes, it is possible to improve image quality by improving image quality by minimizing aberrations and ocular scattering; but it is impossible to exceed the limits of image quality due to diffraction. Both aberrations and ocular scattering, and therefore optical quality of the retinal image are affected by DM [8].

Therefore, diabetic eye screening is an important health policy in worldwide to prevent diabetic retinopathy (DR). Previously, many countries do not practice annual eye checkup for diabetic retinopathy but currently practice annual eye check-up which include Tanzania [9], Korea [10], Spain [11], Yemen, Sri Lanka [12], Canada [13]. But nowadays, most countries are practicing annual eye screening.

In a study by Byun, et al. [10], 1,288 patients were diagnosed with diabetes at $\geq 30$ years of age from 24,871 participants from the Korean National Health and Nutrition Examination Survey. Initiation of this study was due to only $39 \%$ of people with diabetes received a dilated eye examination. Thus, this study was conducted to identify factors that were associated with screening for diabetic retinopathy and nephropathy. In this study, 468 patients were received screening for diabetic retinopathy during previous year.

In Malaysia, two-tiered health care system (government and private sector) was applied. In Diab Care 2008, among 1670 diabetic population, the most common eye complications were cataract $(27.2 \%)$ and non-proliferative retinopathy (22.8\%), whereas severe late eye complications that were reported included photocoagulation (15.3\%), proliferative diabetic retinopathy (13.7\%), advanced eye disease (5.3\%) and blindness (1.7\%). Increase prevalence of DM due to an increase prevalence of overweight and obesity. It was due to poor dietary adherence, high consumption of carbohydrate and sedentary lifestyle and $68 \%$ had eye screening. Thus, multidisciplinary team will be formed to the betterment of managing chronic disease.

In addition, Mafauzy, et al. reported that one-third of patients have a poor quality of life among 1549 diabetic patients in public hospitals across Malaysia. Eye complication was $67.9 \%$ which represented by data on fundus examination was obtained in $55.0 \%$ of the patients found that background retinopathy was present in $11.1 \%$ of the patients; $16.2 \%$ of the patients had cataract; $5.4 \%$ had advanced diabetic eye disease, $10.8 \%$ had photocoagulation done and $0.8 \%$ had legal blindness. In short, there was poor adherence to diet, exercise, and self- testing of blood glucose. 
Thus, self-monitoring is important to emphasize among diabetic patients. A similar view was stated by Daniel, et al. as early detection and prompt treatment allow prevention of diabetes-related visual impairment. It is due to the risk factors of DR that can be broadly divided into modifiable (hyperglycemia, hypertension, hyperlipidemia and obesity) and non-modifiable (duration of disease, puberty, and pregnancy). Modifiable risk factor could be monitored and prevented through proper interventional steps ahead.

In Malaysia, it has been recommended that all Diabetes Mellitus (DM) should have at least yearly eye examination for diabetic retinopathy but according to the National Health and Morbidity Survey (NMHS) III 2006, only 45\% of patients with known DM ever had an eye examination [14]. It was similar as Perera [15] found that only $50 \%$ of study participants knew the importance of assessing vision annually to prevent diabetic retinopathy.

In addition, instruments for eye screening include direct ophthalmoscope, PAN ophthalmoscope, Binocular indirect ophthalmoscope (BIO), slit lamp biomicroscope, mydratic fundus camera and non-mydratic fundus camera. With advancements in technology, diabetic eye screenings are being conducted using digital fundus cameras in Australia, the United States, the United Kingdom and Thailand. Some of these countries employ the telemedicine approach, where the fundus images are sent to grading centers via the internet for grading by ophthalmologists [4]. The grading system is based on International Clinical Diabetic Retinopathy and Diabetic Macula Oedema Disease Severity Scale. There are few guidelines and recommendation for DR eye screening across countries (Table 1). However, there some criteria for urgent referral as below:

\begin{tabular}{|c|c|}
\hline Urgency of Referral & Ocular Features \\
\hline \multirow{2}{*}{ Emergency (same day referral) } & Sudden severe visual loss \\
\cline { 2 - 2 } & Symptoms or signs of acute retinal detachment \\
\hline \multirow{2}{*}{ Within 1 week } & Presence of retinal new vessels \\
\cline { 2 - 2 } & Preretinal haemorrhage \\
\cline { 2 - 2 } & Vitreous haemorrhage \\
\cline { 2 - 2 } & Rubeosis iridis \\
\cline { 2 - 2 } Within 4 week & Any form of maculopathy \\
\cline { 2 - 2 } & Severe NPDR \\
\cline { 2 - 2 } & Worsening retinopathy \\
\cline { 2 - 2 }
\end{tabular}

Table 1: Criteria for urgent referral.

Source: National Institute for Clinical Excellence. Management of Type 2 diabetes. Retinopathy screening and early management London; NICE, [16]

In Malaysia, there are 107 fundus cameras in the health clinic. However, this facility is limited in Pahang as there are nine fundus cameras provided with 14 ophthalmologists (12 persons in public and 2 persons in private); ophthalmologist services is provided in two public hospitals and one private clinic which cover $0.02 \%$ from 10,000 population. Also, there are 12 optometrists in public sector, two assistant Medical officer (MO) with ophthalmology post basic and 9 assistant Medical officers without post basic and 10 nurses with and without ophthalmology post basic equally.

\section{Frequency of Eye Screening}

There are few scholars discussed about decision of diabetic eye screening interval. Soto-Pedre, et al. [11] conducted an observational retrospective follow-up study attending a diabetes center among 508 diabetic patients to validate frequency of eye screening visits. In this study, the median recommendation screening interval was 20 months and screening frequency should be reduced by $40 \%$ compared with fixed annual screening to reduce Sight-Threatening Diabetic Retinopathy (STDR) in diabetic patients in order to increase cost-effectiveness.

Inconsistent with that, Taylor, et al. [17] suggested that extending screening intervals beyond one year or two years have the different benefit. Eye screening interval suggested between every two years could be safe with those with no background or mild retinopathy but six months interval should be applied to retinopathy patient. Besides, many factors to measure cost-effectiveness which include progression rates between disease stages, an interval between screening visits, compliance and sensitivity and specificity of testing. 
In short, eye screening interval safety is still debatable whether biannual (every 6 months), annually, and biennial (every 24 months) in order to reduce risk of STDR. However, lower uptake of eye screening remains an important issue that involves cost-effectiveness analysis in disease management and current evidence does not support a move to extend beyond one-year screening.

\section{Barriers to Uptake Diabetic Eye Screening}

There are many barriers in eye screening. These include personal barrier, financial issue and access, health literacy, health care personnel and guideline implementation.

Personal barriers: Some studies were done to measure the level of awareness of diabetic ocular complication. In a study by Hoque, et al. [18] at Bangladesh, only $4.9 \%$ of their study participants were aware of diabetes eye disease which is lower than study by Bodunde [19]. In Malaysia, high-level awareness of diabetic ocular complication was reported as $86 \%$ [6] and $91.8 \%$ of participants [5]. In a study done in Pahang, found that cataract was the most common eye disease as involved $22.2 \%$ from 1081 patient while diabetic retinopathy was $7.21 \%$ respectively [1]. However, high level of awareness does not necessarily translated into practice. As evidence by National Eye Database (NED), it was reported that the proportion of patients with blindness was 9.0\% [7].

A study conducted by Tajunisah, et al. [6] at University Malaya Medical Centre (UMMC) assessed about awareness of eye complications and the prevalence of retinopathy in the eye clinic among 137 type II diabetic patients who attend tertiary medical center in Kuala Lumpur. It found that 86\% of respondent aware of diabetic eye complications especially who had achieved tertiary educational level (96.3\%). Despite a high level of awareness, only $21.9 \%$ had recorder HBA1c level of $<6.5 \%$ while $31.4 \%$ were under the erroneous assumption of having a good blood sugar control. Many of patients (43.8\%) did not know how frequent they should go for eye checkup and $72.3 \%$ did not know what treatments were available.

A total of $29.2 \%$ had diabetic retinopathy in their first eye testing. Some barriers for not coming for eye checkup include lack of understanding on diabetic eye disease (68.6\%), had other healthcare provider (18.2\%), lack of access to eye care $(5.1 \%)$, time limitations $(4.4 \%)$, cost or insurance issue $(2.9 \%)$ as well as fear of discovering something bad $(0.7 \%)$. This study represents urban population as they have high level of education and further exploration need on barriers on eye seeking behavior and health education issue.

Masliza, et al. studied about predisposing factors for proliferative diabetic retinopathy (PDR) among 120 participants. This study used fundus photography to detect changes and categorized into three groups; no DR, PDR and other findings. It found that, age, duration of disease, nephropathy and peripheral neuropathy were significantly associated with PDR. However, this study used retrospective record review which may increase selection bias.

A study done by Elizabeth stated that lack of patients' health education and knowledge about Diabetes Mellitus and Diabetic Retinopathy as the most significant hindrance. Bodunde, et al. [19] stated that awareness of ocular complications does not necessarily translate into knowledge. Likewise, the insight of disease affects patients' attitude and practice [20,21]. Conversely, knowledge on ocular effect of DM was not related to the level of patients' formal education [20] which reflects that specific education-dependent strategy must be explored.

A study done by Tajunisah, et al. [6] stated that about $48.9 \%$ of participants thought they have good sugar control while $31 \%$ have the wrong impression of having good control and 5.8\% had no idea at all about complication. However, $77.7 \%$ of participants in the study by Bodunde [19] aware that it can affect eyes, 58.8\% did not know which part of the eye that will be affected. Lastly, patients were not coming for eye screening because they had no idea regarding eye checkup as $43.8 \%$ of 148 participants did not know the frequency of eye checkup [6].

Salmiah held interview among diabetes patients and health care provider to explore barriers to optimal control of type 2 Diabetes Mellitus. It used grounded theory method. Many factors contribute to personal barriers include coping strategies and difficulties in integrating regimen into their lifestyle, diabetic health literacy, knowledge about the disease and family support. However, HCP stated that barriers include financial background, self-management blood glucose and perception of disease.

\section{Financial Issue and Access}

Financial issue and accessibility to health care services affect patients' attendance to health care center. It is due to cost of hospital billing, medication, and transportation expense to health care center. Besides, families always contribute in patients' management of disease; acquiring medicine, accompanying the person to health care services, knowledge sharing, and upholding a healthy diet since buying food was a collective matter especially in times of financial hardship. In addition, access to eye screening appointment place a great challenge. It includes geographical location $[1,22]$, multiple appointment systems and long waiting list. It causes a burden for patients and family members for the arrangement to a clinical session. 
Health literacy: In addition, poor health literacy makes diabetic education and self-management not deliberate widely among patients. In the study by Stiles [23] and Ali [24] stated that diabetic education and health literacy is important for diabetic patients to understand the disease process. Well-educated patients always take an empowered role in dealing with health care providers, seeking credit for having knowledge and intelligent before their appointment while less well-educated patients were more submissive.

Healthcare professional: Currently, multiple health care professionals (HCP) perform DR screening which includes an ophthalmologist, physician, screening technician and nurses. Thus, information regarding eye screening could be provided by primary care physician, ophthalmologist, medical officer or nurses during check-up to patients. However, awareness of complication correlated with choice of HCP approach by patients as $81.4 \%$ chose to visit ophthalmologist and more information about complication received [6].

The skill of handling equipment by medical staffs influences grading of eye disease and treatment. For example, fundus examination can be done by using a funduscopy and not necessarily by using a fundus camera. However, skillful staff is needed to ensure good grading and management taken place. As for health care sector, wealth resources are important to maintain the good condition of equipment and facilities. Thus, a cost-effective analysis was done to compare interval of eye screening and annual screening remains the most cost-effective $[11,17]$.

Guideline implementation: Nevertheless, there are many barriers to adhere to diabetic eye screening guideline. It is due to different type of diabetic retinopathy (DR) and duration of disease (5) poor glycemic control, prompt referral of sight-threating DR, lack of optimization of fundus photography, different standard of grading system and also uninsured issue for eye screening.

Furthermore, barriers in applying CPG in health care services include lack of linkages between services and provider, lack of recalls or reminders for defaulters, long waiting list for first screening and referral to see ophthalmologists, assessment of the implementation of screening programs that are based on this approach to confirm its clinical cost-effectiveness [11] and effectiveness of communication about policy changes in institution.

\section{Conclusion}

From the literature review, it is clear that diabetes eye screening remains as an important issue to prevent STDR among diabetes mellitus patients [25]. It was understood that health care facilities have equipped with facilities for diabetic eye screening and health care providers were prepared in every aspect to educate and inculcate awareness on eye screening [26-28]. However, lower uptake of eye screening plays a major question in health care practices. Thus, cost-effectiveness analysis places an important measure to evaluate current practice in this area.

In the review, various patients' perspective towards barriers and support on eye screening were disclosed. Many studies discussed that support system such as family, financial support, and geographical area influences their decision of eye screening because of this place as consideration in their decision making $[29,30]$. In addition, their sociodemographic background also may affect insight, their acceptance, and perspective towards the disease. Thus, by including these three elements; health care system, health care provider and patients in this review can provide a clear explanation of factors influencing the decision for diabetic eye screening.

\section{Limitations of This Review}

This article uses a systematic approach but did not apply a systematic review procedure.

\section{Acknowledgement}

We would like to express our appreciation to International Islamic University Malaysia (IIUM) for funding this study (RIGS16-140-0304).

\section{References}

1. Thevi T, Basri M, Reddy SC (2012) Prevalence of eye diseases and visual impairment among the rural population - A case study of Temerloh Hospital. Malays Fam Physician 7(1):6-10.

2. Goh PP, Hussein E, Ismail M, Mustari Z, Ismail M (2008) The first annual report of the National Eye Database, 2007. Kuala Lumpur: National Eye Database, pp: 1-106.

3. Goh PP, Livingstone B, Hussein E, Ismail M (2010) The second annual report of the National Eye Database, 2008. Kuala Lumpur: National Eye Database, pp: 1-144.

4. Goh PP, Omar MA, Yusoff AF (2010) Diabetic eye screening in Malaysia: findings from the National Health and Morbidity Survey 2006. Singapore Med J 51(8): 631634.

5. Addoor KR, Bhandary SV, Khanna R, Rao LG, Lingam KD, et al. (2011) Assessment of awareness of diabetic retinopathy among the diabetics attending the peripheral diabetic clinics in Melaka, Malaysia. Med J Malaysia 66(1): 48-52. 
6. Tajunisah I, Wong PS, Tan LT, Rokiah P, Reddy SC (2011) Awareness of eye complications and prevalence of retinopathy in the first visit to eye clinic among type 2 diabetic patients. Int J Opthalmol 4(5): 519-524.

7. Ministry of Health (2011) Clinical practice guidelines: Screening of diabetic retinopathy. Putrajaya (Malaysia): CPG Secretariat.

8. Calvo Maroto AM, Perez CAmbrodi RJ, Albaran Diego C, Pons A, Cervino A (2014) Optical quality of the diabetic eye: A review. Eye 28(11): 1271-1280.

9. Omar FJ, Sheeladevi S, Rani PK, Ning G, Kabona G (2014) Evaluating the effectiveness of opportunistic eye screening model for people with diabetes attending diabetes clinic at Mnazi Mmoja hospital, Zanzibar. BMC Ophthalmol 14(1): 81-87.

10. Byun SH, Ma SH, Jun JK, Jung KW, Park B (2013) Screening for diabetic retinopathy and nephropathy in patients with diabetes: A nationwide survey in Korea. PLoS ONE 8(5): e62991.

11. Soto Pedre E, Hernaez Ortega MC, Pinies JA (2007) Duration of diabetes and screening coverage for retinopathy among patients with type 2 diabetes. Ophthalmic Epidemiol 14(2): 76-79.

12. Katulanda P, Ranasinghe P, Jayawardena R, Sheriff R, Matthews DR (2015) The influence of family history of diabetes on disease prevalence and associated metabolic risk factors among Sri Lankan adults. Diabet Med 32(3): 314-323.

13. Kiran T, Kopp A, Moineddin R, Victor JC, Campbell RJ, et al. (2013) Unintended consequences of delisting routine eye exams on retinopathy screening for people with diabetes in Ontario, Canada. CMAJ 185(3): E167-E173.

14. Chan YY, Teh CH, Lim KK, Lim KH, Yeo PS, et al. (2015) Lifestyle, chronic diseases and self-rated health among Malaysian adults: Results from the 2011 National Health and Morbidity Survey (NHMS). BMC Public Health 15: 754-765.

15. Perera DP, De Silva REE, Perera, WLSP (2013) Knowledge of diabetes among type 2 diabetes patients attending a primary health care clinic in Sri Lanka. East Mediterr Health J 19(7): 644-648.

16. National Institute for Clinical Excellence (2002) Clinical Guideline E: Management of Type 2 diabetes. Retinopathy-screening and early management. London: National Institute for Clinical Excellence, pp: 1-15.

17. Taylor Phillips S, Mistry H, Leslie R, Todkill D, Tsertsvadze
A, et al. (2016) Extending the diabetic retinopathy screening interval beyond 1 year: Systematic review. Br J Ophthalmol 100(1): 105-114.

18. Hoque MA, Islam M, Khan M, Ahasan H (2009) Knowledge of diabetic complications in a diabetic population. J Medicine 10(2): 90-93.

19. Bodunde OT, Odusan O, Ogunsemi O, Ajibode HA, Raimi TH (2014) Awareness of ocular complications among diabetic patients in a tertiary hospital in Western, Nigeria. IOSR J Dent Med Sci 13(6): 9-12.

20. Khandekar R, Al Harby S, Al Lawarati J (2010) Knowledge, attitude and practice regarding eye complication and care among Omani persons with diabetes - A cross sectional study. Oman J Opthalmol 3(2): 60-65.

21. Nsiah Kumi P, Ortmeier SR, Brown AE (2009) Disparities in diabetic retinopathy screening and disease for racial and ethnic minority populations-A literature review. J Natl Med Assoc 101(5): 430-437.

22. Lee DJ, Kumar N, Feuer WJ, Chou CF, Rosa PR, et al. (2014) Dilated eye examination screening guideline compliance among patients with diabetes without a diabetic retinopathy diagnosis: The role of geographic access. BMJ Open Diabetes Res Care 2(1): e000031.

23. Stiles E (2011) Promoting health literacy in patients with diabetes. Nursing Standard 26(8): 35-41.

24. Ali SM, Jusoff K (2009) Barriers to optimal control of type 2 diabetes in Malaysian Malay patients. Glob J Health Sci 1(2): 106-118.

25. Dorner TE, Lackinger C, Schindler K, Stein KV, Rieder A, et al. (2013) Health information regarding diabetes mellitus reduces misconceptions and underestimation of consequences in the general population. Public Health Nutr 16(11): 2032-2039.

26. Bamashmus MA, Gunaid AA, Khandekar RB (2009) Diabetic retinopathy, visual impairment and ocular status among patients with diabetes mellitus in Yemen: A hospital-based study. Indian J Ophthalmol 57(4): 293298.

27. Hartnett ME, Key IJ, Loyacano NM, Horswell RL, Desalvo KB (2005) Perceived barriers to diabetic eye care: Qualitative study of patient and physicians. Arch Ophthalmol 123(3): 387-391.

28. Kumar PJ, Clark ML (2005) Clinical medicine $6^{\text {th }}$ (Edn.), Edinburgh (New York): WB Saunders.

29. Letchuman GR, Wan Nazaimoon WM, Wan Mohamad 
WB, Chandran LR, Tee GH, et al. (2010) Prevalence of diabetes in the Malaysian National Health Morbidity Survey III 2006. Med J Malaysia 65(3): 180-186.
30. Raman R, Gella L, Srinivasan S, Sharma T (2016) Diabetic retinopathy: An epidemic at home and around the world. Indian J Opthalmol 64(1): 69-75. 